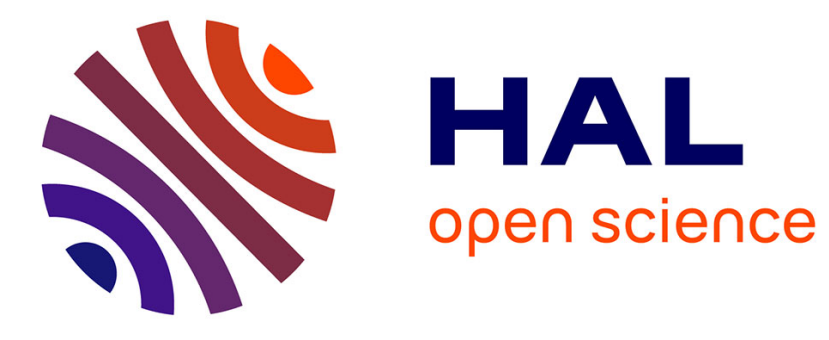

\title{
Hyperspectral image analysis using multifractal attributes
}

Sébastien Combrexelle, Herwig Wendt, Jean-Yves Tourneret, Stephen Mclaughlin, Patrice Abry

\section{- To cite this version:}

Sébastien Combrexelle, Herwig Wendt, Jean-Yves Tourneret, Stephen Mclaughlin, Patrice Abry. Hyperspectral image analysis using multifractal attributes. 7th IEEE Workshop on Hyperspectral Image and SIgnal Processing: Evolution in Remote Sensing (WHISPERS 2015), Jun 2015, Tokyo, Japan. pp. 1-4. hal-01511880

\author{
HAL Id: hal-01511880 \\ https://hal.science/hal-01511880
}

Submitted on 21 Apr 2017

HAL is a multi-disciplinary open access archive for the deposit and dissemination of scientific research documents, whether they are published or not. The documents may come from teaching and research institutions in France or abroad, or from public or private research centers.
L'archive ouverte pluridisciplinaire HAL, est destinée au dépôt et à la diffusion de documents scientifiques de niveau recherche, publiés ou non, émanant des établissements d'enseignement et de recherche français ou étrangers, des laboratoires publics ou privés. 


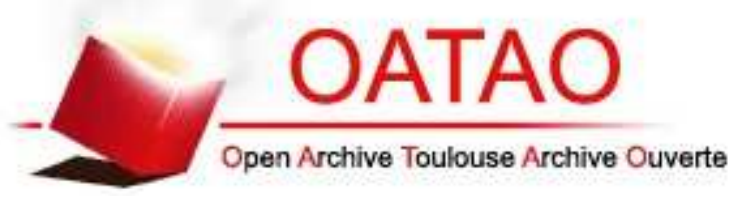

\section{Open Archive TOULOUSE Archive Ouverte (OATAO)}

OATAO is an open access repository that collects the work of Toulouse researchers and makes it freely available over the web where possible.

This is an author-deposited version published in : http://oatao.univ-toulouse.fr/ Eprints ID : 17041

The contribution was presented at WHISPERS 2015 :

http://www.ieee-whispers.com/index.php/past-editions/2015-tokyo-japan

To cite this version : Combrexelle, Sébastien and Wendt, Herwig and Tourneret, Jean-Yves and Mclaughlin, Stephen and Abry, Patrice Hyperspectral image analysis using multifractal attributes. (2015) In: 7th IEEE Workshop on Hyperspectral Image and SIgnal Processing: Evolution in Remote Sensing (WHISPERS 2015), 2 June 2015 - 5 June 2015 (Tokyo, Japan).

Any correspondence concerning this service should be sent to the repository administrator: staff-oatao@listes-diff.inp-toulouse.fr 


\title{
HYPERSPECTRAL IMAGE ANALYSIS USING MULTIFRACTAL ATTRIBUTES
}

\author{
Sébastien Combrexelle ${ }^{1}$, Herwig Wendt ${ }^{1}$, Jean-Yves Tourneret ${ }^{1}$, Stephen McLaughlin ${ }^{2}$ and Patrice Abry ${ }^{3}$ \\ ${ }^{1}$ University of Toulouse, IRIT/INP-ENSEEIHT, Toulouse, France \\ ${ }^{2}$ Heriot-Watt University, School of Engineering and Physical Sciences, Edinburgh, UK \\ ${ }^{3}$ Ecole Normale Supérieure de Lyon, Physics Dept., CNRS, France
}

\begin{abstract}
The increasing spatial resolution of hyperspectral remote sensors requires the development of new processing methods capable of combining both spectral and spatial information. In this article, we focus on the spatial component and propose the use of novel multifractal attributes, which extract spatial information in terms of the fluctuations of the local regularity of image amplitudes. The novelty of the proposed approach is twofold. First, unlike previous attempts, we study attributes that efficiently summarize multifractal information in a few parameters. Second, we make use of the most recent developments in multifractal analysis: wavelet leader multifractal formalism, the current theoretical and practical benchmark in multifractal analysis, and a novel Bayesian estimation procedure for one of the central multifractal parameters. Attributes provided by these stateof-the-art multifractal analysis procedures are studied on two sets of hyperspectral images. The experiments suggest that multifractal analysis can provide relevant spatial/textural attributes which can in turn be employed in tasks such as classification or segmentation.
\end{abstract}

Index Terms - Hyperspectral imaging, multifractal analysis, spatial information, texture characterization, wavelet leaders

\section{INTRODUCTION}

Hyperspectral (HS) imaging is a remote sensing technique which has sparked considerable interest over the last decades. It consists of acquiring an image of a scene in many narrow contiguous spectral bands. This relative wealth of spectral information has motivated the development of processing methods that focus on the spectral information on a pixel-by-pixel-level while neglecting the spatial information contained in the image. However with recent advances in HS remote sensor development, achieving sub-meter spatial resolution, efficiently extracting the spatial information and including it into processing schemes has been recognized as an important topic.

A number of studies suggests that the combination of both spectral and spatial information can improve the performance in classical HS image processing tasks, such as classification [1,2], segmentation [3] or endmember identification [4,5]. Several authors have proposed to extract spatial information by using textural characterizations, see, e.g., $[2,5]$. The notion of image texture does not rely on one single concept, and different paradigms have been proposed in the literature [6]. Among them, scale invariance and multifractal analysis have been reported to be relevant for the effective modeling of textures associated with natural images [7]. The notion of scale invariance is deeply tied to the spatial fluctuations of the pointwise singular behavior of the image amplitudes, measured by the Hölder exponent [8]. Multifractal analysis provides both the theoretical and the practical framework for its study. It achieves a rich yet global description of the spatial fluctuations in terms of the multifractal spectrum. It also provides a formula for its practical estimation, essentially relating the multifractal spectrum to the power law behavior across scales of the moments of multiresolution (wavelet) coefficients associated with the texture [9].

In the context of HS image processing, several attempts have been reported for the use of fractal and multifractal concepts, see, e.g., $[10,11]$ for two recent contributions. While these results are encouraging, most of the attempts remain limited for two reasons: conceptually because they only consider fractal dimensions instead of the richness of a full multifractal characterization; practically because the algorithms traditionally associated with this quantity (essentially, the box-counting algorithm) are limited in accuracy.

The goal of this contribution is to study the use of attributes derived from the multifractal spectrum in the context of HS image processing, making use of the most recent developments in multifractal analysis. To this end, we base our analysis on the current benchmark multifractal formalism, the wavelet leader multifractal formalism (see, e.g., [7] for details on its use for image texture characterization). Secondly, we make use of original multifractal attributes, the log-cumulants, which efficiently capture the main features of the multifractal spectrum with a few parameters. Finally, we emphasize the use of one specific attribute, the multifractality parameter, for which a versatile and powerful Bayesian estimator has recently been introduced $[12,13]$. To our knowledge, such an analysis has never been reported before in the HS imaging context.

The remainder of this article is organized as follows. Section 2 presents the main theoretical and practical concepts of multifractal analysis for HS images and introduces the state-of-the-art estimation procedures. Section 3 describes the analysis of two HS images using multifractal attributes. Conclusions and perspectives for future research are outlined in section 4.

\section{MULTIFRACTAL ANALYSIS OF HS IMAGES}

Within this article we propose to apply multifractal analysis band by band. This amounts to considering each subband as a surface whose regularity is characterized by multifractal analysis of images. Theoretical and practical concepts are recalled below.

Multifractal spectrum. Multifractal analysis studies an image $X: \mathbb{R}^{2} \rightarrow \mathbb{R}$ through the fluctuations (with respect to space) of the local regularity of image amplitudes, commonly measured by the Hölder exponent: the image $X$ is said to belong to $C^{\alpha}\left(t_{0}\right)$ if there exists $\alpha>0$ and a polynomial $P_{t_{0}}$ of degree smaller than $\alpha$ such that $\left\|X(\boldsymbol{t})-P_{\boldsymbol{t}_{0}}(\boldsymbol{t})\right\| \leq C\left\|\boldsymbol{t}-\boldsymbol{t}_{0}\right\|^{\alpha}$ and the Hölder exponent at position $\boldsymbol{t}_{0} \in \mathbb{R}^{2}$ is the largest value of $\alpha$ such that this inequality holds, i.e., $h\left(\boldsymbol{t}_{0}\right) \triangleq \sup \left\{\alpha: X \in C^{\alpha}\left(\boldsymbol{t}_{0}\right)\right\}$. Qualitatively, the Hölder exponent measures the roughness of the image around $t_{0}$ (the smaller $h\left(t_{0}\right)$, 
the rougher $X$ at $t_{0}$; the larger $h$, the smoother the image). The textural information is then extracted from the spatial fluctuations of the Hölder exponent. Multifractal analysis provides a measure for these regularity fluctuations by means of the multifractal spectrum $D(h)$. It is defined as the Hausdorff dimension (denoted $\operatorname{dim}_{H}$ ) of the sets of points at which the Hölder exponent takes the same value

$$
D(h) \triangleq \operatorname{dim}_{H}\{\boldsymbol{t}: h(\boldsymbol{t})=h\}
$$

i.e., $D(h)$ measures the "size" of the set of points having the same roughness. It can be shown that the multifractal spectrum admits the following polynomial expansion around its maximum [7]

$$
D(h)=2+\frac{c_{2}}{2 !}\left(\frac{h-c_{1}}{c_{2}}\right)^{2}-\frac{c_{3}}{3 !}\left(\frac{h-c_{1}}{c_{2}}\right)^{3}+o\left(\left(h-c_{1}\right)^{3}\right)
$$

where $o\left(x^{3}\right) / x^{3}$ tends to zero when $x$ tends to 0 . The coefficients $c_{p}$ in this expansion are termed the log-cumulants. The leading order coefficients provide an efficient summary of the multifractal properties of $X$ and are often used in applications instead of the full function $D(h): c_{1}$ corresponds to the mode of $D(h)$ ("average" smoothness), $c_{2}$ is directly related to its width, and $c_{3}$ to the asymmetry.

Wavelet leaders multifractal formalism. The mathematical definition of $D(h)$ cannot be used for its practical estimation. Instead, a so-called multifractal formalism can be constructed based on specific multiresolution coefficients $\ell(j, \boldsymbol{k})$, the wavelet leaders [7,9], that capture the local regularity of the image $X$ around the discrete spatial location $\boldsymbol{k}$ for a given frequency scale $a=2^{j}$. Let $D_{X}^{(m)}(j, \boldsymbol{k}), m=0, \ldots, 3$ denote the coefficients of a $2 \mathrm{D}$ dyadic wavelet transform, defined by a mother wavelet $\psi$ characterized by its number $N_{\psi}$ of vanishing moments, see, e.g., [14] for details. For multifractal analysis purposes, the wavelet coefficients are renormalized to the $L^{1}$-norm, i.e., $d_{X}^{(m)}(j, \boldsymbol{k}) \triangleq 2^{-j / 2} D_{X}^{(m)}(j, \boldsymbol{k}), \quad m=$ $1,2,3$. The wavelet leaders $\boldsymbol{\ell}(j, \boldsymbol{k})$ are then defined as the local supremum of wavelet coefficients taken within this spatial neighborhood and over all finer scales: Let denote $\lambda_{j, k}=\left\{\left[k_{1} 2^{j},\left(k_{1}+\right.\right.\right.$ 1) $\left.\left.2^{j}\right),\left[k_{2} 2^{j},\left(k_{2}+1\right) 2^{j}\right)\right\}$ the dyadic cube of side length $2^{j}$ centered at $\boldsymbol{k} 2^{j}$ and $3 \lambda_{j, \boldsymbol{k}}=\bigcup_{n_{1}, n_{2}=\{-1,0,1\}} \lambda_{j, k_{1}+n_{1}, k_{2}+n_{2}}$ the union of this cube with its eight neighbors, then [7,9]

$$
\boldsymbol{\ell}(j, \boldsymbol{k}) \triangleq \sup _{m \in(1,2,3), \lambda^{\prime} \subset 3 \lambda_{j, \boldsymbol{k}}}\left|d_{X}^{(m)}\left(\lambda^{\prime}\right)\right|
$$

The multifractal formalism essentially states that the log-cumulants $c_{p}$ in (1) can be directly related to the wavelet leaders $\ell(j, k)$ through the key relation

$$
C_{p}(j)=c_{p}^{0}+c_{p} \log 2^{j}
$$

where $C_{p}(j)$ is the $p$-th order cumulant of wavelet leaders $\boldsymbol{\ell}(j, \boldsymbol{k})$, see the seminal contribution [15].

Estimation. The relation (3) leads to the definition of estimators for the parameter $c_{p}$ based on linear regressions of the sample cumulant of $\log \boldsymbol{\ell}(j, \boldsymbol{k})$, denoted by $\widehat{C}_{p}(j)$, against scale $j$ for a range of scales $j \in\left[j_{1}, j_{2}\right]$

$$
\hat{c}_{p}=\frac{1}{\log 2} \sum_{j=j_{1}}^{j_{2}} w_{j} \widehat{C}_{p}(j)
$$

where $w_{j}$ are appropriate regression weights [7]. These expressions are the most frequently used estimators for multifractal attributes.

An alternative estimation procedure for parameter $c_{2}$, based on a Bayesian framework, has been recently proposed, see [12,13]. The parameter $c_{2}$ is of central importance since it enables the identification of the two most important process model classes used in applications, namely self-similar processes and multifractal multiplicative cascade based processes (corresponding to $c_{2}=0$ and $c_{2}<0$, respectively). The Bayesian approach relies on a semi-parametric multivariate Gaussian model for $\log$-leaders $\log \ell(j, \boldsymbol{k})$ that efficiently exploits their covariance structure in addition to the relation (4) for the evolution of their variance across scales. This approach has been shown to yield significantly improved performance and enables the accurate multifractal analysis of small image patches. Furthermore, it can be incorporated in a hierarchical statistical model in order to account for the dependencies between image patches, for instance. The main drawback of this approach as compared to the use of (4) is the fact that the image patch size can not be smaller than $64 \times 64$ and its increased computational cost, ranging from $\sim 8 \mathrm{~s}(64 \times 64)$ to $\sim 50$ s $(512 \times 512)$.

To summarize, the multifractal analysis of an image consists, first, in the computation of wavelet leaders, second, in the computation of sample cumulants and estimation of log-cumulants.

\section{MULTIFRACTAL ATTRIBUTES FOR HS IMAGES}

Data sets. To assess the relevance of $\log$-cumulants $c_{p}$ in the context of HS image processing, two sets of HS images are used. The first HS image was acquired during the Madonna project [16] over Villelongue (France) by the Hyspex hyperspectral sensor providing measurements from the visible to near infrared bands with a spatial resolution of 0.5 meters. It is mainly composed of forested and urban areas as shown in Fig. 1(a) (artificial color composition). The second image was acquired over Moffett Field (CA, USA) by the Airborne Visible Infrared Imaging Spectrometer AVIRIS of the JPL laboratory (NASA) with a spatial resolution around 20 meters (artificial color composition shown in Fig.1(b)).

Estimation. We use tensor-products of Daubechies's mother wavelet with $N_{\psi}=2$ vanishing moments in the $2 \mathrm{D}$ wavelet transform. The weights $w_{j}$ in (4) are chosen proportional to the number of wavelet leaders at scale $j$, as in [7], to reflect the confidence granted to $\widehat{C}_{p}(j)$. In the Bayesian estimation procedure, a bandwidth parameter $\eta$ controls the percentage of the spectral grid used to approximate the Gaussian likelihood and is set to $\eta=0.3$, see [13]. Scales used to perform estimation are respectively $\left[j_{1}, j_{2}\right]=[2,4]$, $[1,2]$ and $[1,3]$ for patches of size $256 \times 256,64 \times 64$ and $16 \times 16$ pixels.

\subsection{Spectral evolution of multifractal features}

We begin with investigating the evolution of multifractal image properties across spectral bands. For this purpose, the estimation procedures described in Section 2 are applied independently to each subband of a homogenous area of $256 \times 256$ pixels of the Moffet data set (the mountain area indicated by a red frame in Fig. 1).

Figure 2 (b) plots multifractal spectra of the image 2 (a) for three different bands. The spectra for different bands clearly differ in shape and position. The mode, the width and the asymmetry of the spectra (tied to $c_{1}, c_{2}$ and $c_{3}$ ) have their own independent spectral evolutions. For instance, bands \# 20 and 96 have similar values of $c_{1}$ but different values of $c_{3}$.

We propose to investigate in more detail the spectral evolution of the second $\log$-cumulant $c_{2}$, which can be estimated by least squares (linear regression (4)) or the Bayesian approach, allowing further discussion. The spectral evolution of $c_{2}$ is displayed in Fig. 2 (c) (blue: Least Squares (LS); red: Bayesian estimation (Bay)). For 


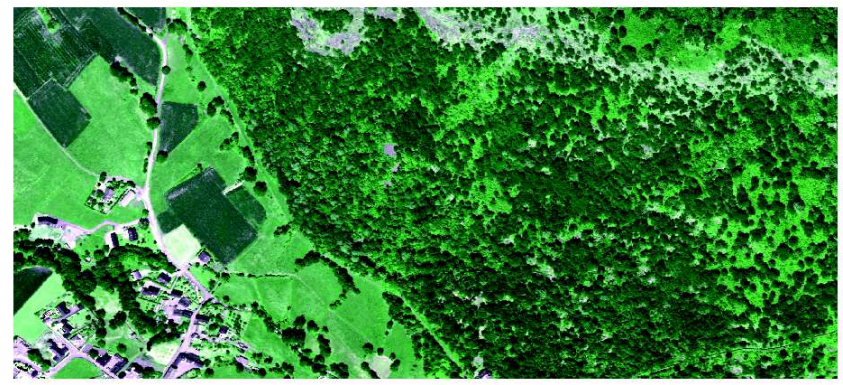

(a) Madonna

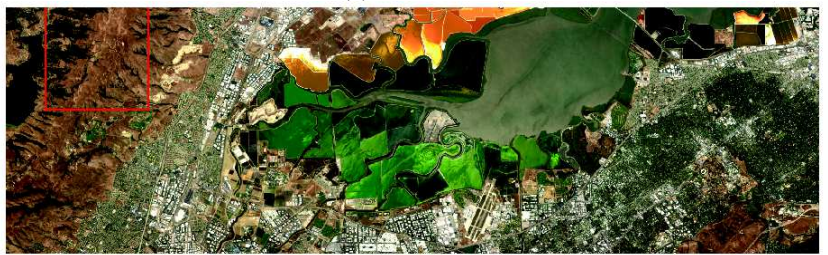

(b) Moffett

Fig. 1. Real HS data acquired by the Hyspex hyperspectral scanner (a) and by AVIRIS (b).

comparison, the average reflectance of the image 2 (a) versus spectral bands is reproduced in Fig. 2 (d) with its standard deviation (STD) (red: average reflectance; blue: standard deviation). The evolution of the multifractal attribute $c_{2}$ across spectral bands significantly differs from the average reflectance, providing clear evidence for the complementary information that is captured by multifractal texture analysis. The correlation coefficient between the reflectance and $c_{2}$ is found to be small: $0.1(0.4)$ for the average reflectance and $-0.7(-0.5)$ for the standard deviation when using Bayesian estimator (LS estimator), respectively. In addition, we observe a particularly pronounced fluctuation of $c_{2}$ around subbands 10 and 30 that cannot be identified on the reflectivity spectrum.

These results suggest that the log-cumulants are local spatial attributes whose spectral evolution significantly differs from that of the average reflectivity and hence convey complementary information.

\subsection{Spatial distribution of multifractal features}

A second set of experiments is conducted on small patches for a single subband of the HS images in order to illustrate and study the description enabled by the multifractal attributes throughout space.

In a first experiment, the patch size is set to the minimum value allowed by the Bayesian approach $(64 \times 64)$, and $\log$-cumulants $c_{2}$ are estimated for half-overlapping patches of the reflectivity image for two different subbands independently. The results are summarized in Figs. 3 and 4 for Moffet and Madonna: reflectivity image (top row), LS estimator (second row) and Bayesian estimator (bottom row) for two different bands (left and right column, respectively). The LS based estimation is dominated by the Bayesian approach, which manages to reproduce the spatial structure of the image texture with less spatial variability. The $\log$-cumulant $c_{2}$ nicely reproduces the overall spatial composition of the image. Yet, the map of $c_{2}$ does not identically reproduce the original reflectivity image. Indeed, the mountain regions (top-left and bottom-right corners) in Moffett exhibit low contrast with urban areas, while the contrast is particularly pronounced for $c_{2}$. The same comment applies to the path separating the forest and the fields in Madonna. Finally, when comparing the maps of $c_{2}$ for two different bands of Madonna, estimates of the forested area are found to be spatially homogeneous

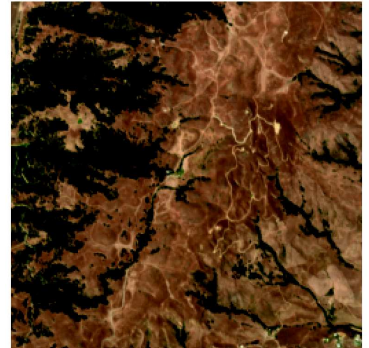

(a) Mountain zone of interest

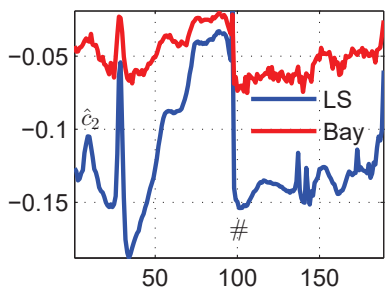

(c) Evolution of $c_{2}$

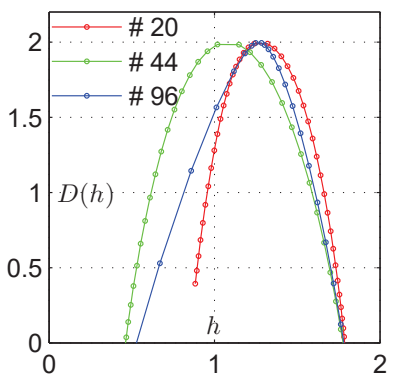

(b) Multifractal spectrum

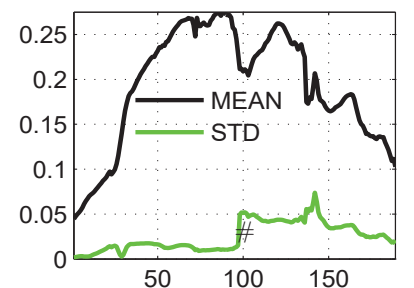

(d) Average reflectance
Fig. 2. Mountain area from Moffett data (a), multifractal spectrum for three different bands, spectral evolution of $c_{2}$ (c) and average reflectance and standard deviation (d).

for the band \# 20 (a). In contrast, clusters of strongly negative $c_{2}$ appear in the forest at band \# 44 (b). This difference apparently reflects a change of the tree density, which affects the width of $D(h)$.

In a second experiment, we reduce the patch size in an attempt to further improve spatial localization and study the behavior of cumulants $C_{p}(j)$ and log-cumulants $c_{p}$, estimated by using (4), for several orders $p$. Estimation is performed using sliding windows of size $16 \times 16$ pixels; thus, each window (patch) can be identified with one pixel (here, the central pixel of the window). Only results for $p=1$ are reproduced here for space reasons. Note that the computation time of this procedure is of the order of only $5 \mathrm{~s}$ for all pixels of a band. Fig. 5 displays the first cumulant $C_{1}(1)$ (top) and the first $\log$-cumulant $c_{1}$ (bottom) for the band \# 44 of the Moffett image. The first cumulant $C_{1}(j)$ can be interpreted as a local energy of the reflectivity image gradient at scale $j$; as such, it can be related to other approaches for computing the (local) energy of the image. The multifractal framework, however, provides a different description by considering the mechanism that relates $C_{p}(j)$ at different scales, encoded by the log-cumulants $c_{p}$. Indeed, the local estimates of $c_{1}$ depicted in Fig. 5 (bottom) suggest the following remarks. Certain types of building groups (center-right part of image), for instance, are highlighted in the map of $c_{1}$ while they were not visible in the original reflectivity image. Along the same line, in the mountain area (upper left corner), the $c_{1}$ map reproduces structures different from the ones in the $C_{1}(1)$ map or even from the reliefs in the intensity map. Finally, borders between water and land, which are in essence discontinuities, are outlined by large values of $c_{1}$.

The results presented above are positive indications suggesting the use of multifractal attributes $c_{p}$ for the extraction of spatial information, not captured by a pixel-by-pixel analysis of the reflectivity image nor by classical processing methods such as local energy.

\section{CONCLUSIONS AND PERSPECTIVES}

This paper has proposed to extract spatial information using multifractal attributes, the log-cumulants $c_{p}$. Experiments, conducted on two HS images, have shown that these log-cumulants captured infor- 


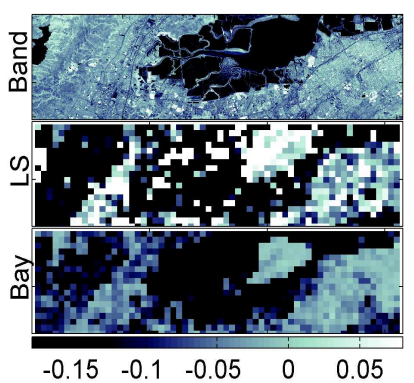

(a) Band \# 44

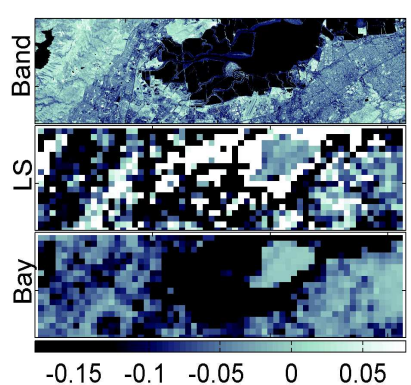

(b) Band \# 96
Fig. 3. Reflectivity image (top), LS (middle) and Bayesian (bottom) estimates of $c_{2}$ for $64 \times 64$ overlapping patches on Moffett data set for bands \# 44 (a) and \# 96 (b).

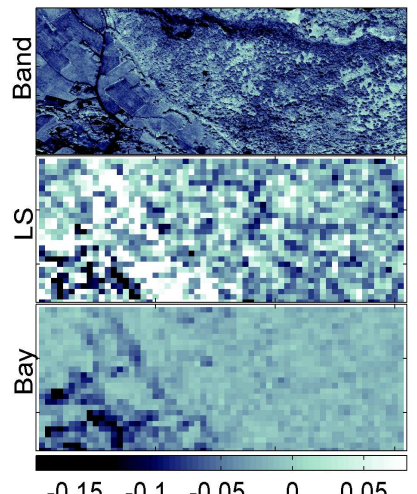

$\begin{array}{lllll}-0.15 & -0.1 & -0.05 & 0 & 0.05\end{array}$

(a) Band \# 20

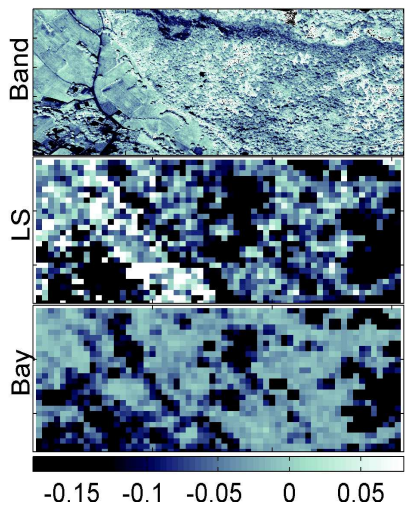

(b) Band \# 44
Fig. 4. Reflectivity image (top), LS (middle) and Bayesian (bottom) estimates of $c_{2}$ for $64 \times 64$ overlapping patches on Madonna data set for bands \# 20 (a) and \# 44 (b).

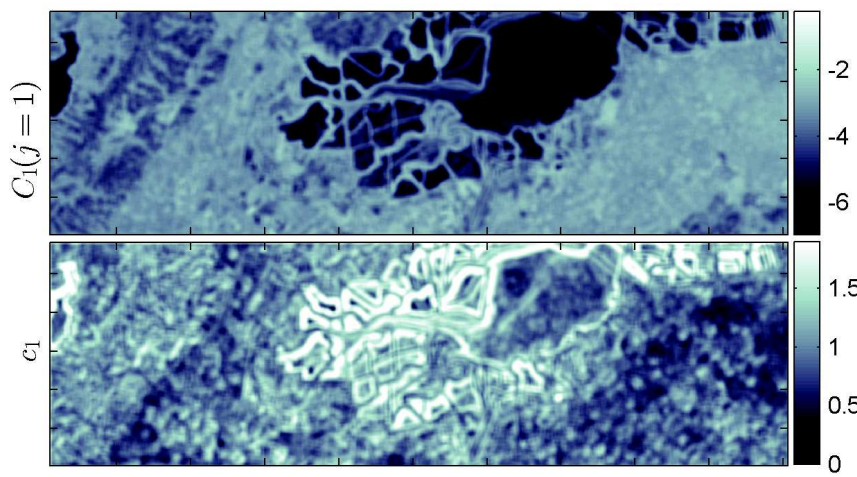

Fig. 5. Pixel-wise map of the first order cumulant $C_{1}(1)$ (top) and $\log$-cumulant $c_{1}$ (bottom) on Moffett data set for band \# 44 .

mation significantly different from the average reflectivity or from the local energy. Viewed as a function of wavelength, they could be incorporated in a data-mining scheme to identify bands of interest based on characteristics of multifractal spectrum (mode, width or asymmetry). Viewed as a function of space, they are attributes characterizing the local texture and could be combined with spectral features to perform tasks such as classification or segmentation. This approach is currently under investigation. Future work on the Bayesian approach will include the definition of hierarchical models to introduce spatial/spectral dependence between $c_{2}$ of different patches and different bands. The statistical model could be enhanced to incorporate $c_{1}$ and $c_{3}$, associated with the mode and skewness of the multifractal spectrum.

\section{ACKNOWLEDGEMENTS}

S. McLaughlin acknowledges the support of EPSRC grant number EP/J015180/1. This work was partially supported by ANR BLANC 2011 AMATIS BS0101102 and ANR Project Hypanema ANR-12BS03-003. S. Combrexelle was supported by the Direction Générale de l'Armement (DGA).

\section{REFERENCES}

[1] M. Fauvel, Y. Tarabalka, J. A. Benediktsson, J. Chanussot, and J. C. Tilton, "Advances in spectral-spatial classification of hyperspectral images.," Proc. IEEE, vol. 101, no. 3, pp. 652-675, 2013.

[2] G. Roussel, V. Achard, A. Alakian, and J.-C. Fort, "Benefits of textural characterization for the classification of hyperspectral images," in Proc. IEEE Workshop Hyperspectral Image Signal Process.: Evolution in Remote Sens. (WHISPERS), Reykjavik, Iceland, June 2010, pp. 1-4.

[3] N. Gorretta, J. M. Roger, G. Rabatel, V. Bellon-Maurel, C. Fiorio, and C. Lelong, "Hyperspectral image segmentation: The butterfly approach," in Proc. IEEE Workshop Hyperspectral Image Signal Process.: Evolution in Remote Sens. (WHISPERS), Grenoble, France, Aug. 2009, pp. 1-4.

[4] G. Martin and A. Plaza, "Spatial-spectral preprocessing prior to endmember identification and unmixing of remotely sensed hyperspectral data," IEEE J. Sel. Top. Appl. Earth Observat. Remote Sens., vol. 5, no. 2, pp. 380-395, April 2012.

[5] M. Zortea, D. Tuia, F. Pacifici, and A. Plaza, "Spectral-textural endmember extraction," in Proc. IEEE Workshop Hyperspectral Image Signal Process.: Evolution in Remote Sens. (WHISPERS), Reykjavik, Iceland, June 2010, pp. 1-4.

[6] R. M. Haralick, "Statistical and structural approaches to texture," Proc. of the IEEE, vol. 67, no. 5, pp. 786-804, 1979.

[7] H. Wendt, S. G. Roux, S. Jaffard, and P. Abry, "Wavelet leaders and bootstrap for multifractal analysis of images," Signal Process., vol. 89, no. 6 , pp. $1100-1114,2009$.

[8] B. B. Mandelbrot, "Intermittent turbulence in self-similar cascades: divergence of high moments and dimension of the carrier," J. Fluid Mech., vol. 62, pp. 331-358, 1974.

[9] S. Jaffard, "Wavelet techniques in multifractal analysis," in Fractal Geometry and Applications: A Jubilee of Benoît Mandelbrot, Proc. Symp. Pure Math., M. Lapidus and M. van Frankenhuijsen, Eds. 2004, vol. 72(2), pp. 91-152, AMS.

[10] P. Dong, "Fractal signatures for multiscale processing of hyperspectral image data," Adv. Space Res., vol. 41, no. 11, pp. 1733 - 1743, 2008.

[11] W. Sun, G. Xu, P. Gong, and S. Liang, "Fractal analysis of remotely sensed images: A review of methods and applications," Int. J. Remote Sens., vol. 27, pp. 4963-4990, 2006.

[12] H. Wendt, N. Dobigeon, J.-Y. Tourneret, and P. Abry, "Bayesian estimation for the multifractality parameter," in Proc. IEEE Int. Conf. Acoust., Speech, and Signal Process. (ICASSP), Vancouver, Canada, May 2013

[13] S. Combrexelle, H. Wendt, N. Dobigeon, J.-Y. Tourneret, S. McLaughlin, and P. Abry, "Bayesian estimation of the multifractality parameter for image texture using a Whittle approximation," arXiv preprint arXiv: 1410.4871, 2014.

[14] S. Mallat, A Wavelet Tour of Signal Processing, Academic Press, 3rd edition, 2008.

[15] B. Castaing, Y. Gagne, and M. Marchand, "Log-similarity for turbulent flows?", Physica D, vol. 68, no. 34, pp. 387 - 400, 1993

[16] D. Sheeren, M. Fauvel, S. Ladet, A. Jacquin, G. Bertoni, and A. Gibon, "Mapping ash tree colonization in an agricultural mountain landscape: Investigating the potential of hyperspectral imagery," in Proc. IEEE Int. Conf. Geosci. Remote Sens. (IGARSS), Vancouver, Canada, July 2011, pp. 3672-3675. 